\title{
BMJ Open Sipjeondaebo-tang in patients with cancer with anorexia: a protocol for a pilot, randomised, controlled trial
}

\author{
Chunhoo Cheon, ${ }^{1}$ Sunju Park, ${ }^{2}$ Yu Lee Park, ${ }^{1}$ Ching-Wen Huang, ${ }^{1}$ Youme Ko, ${ }^{1}$ \\ Bo-Hyoung Jang, ${ }^{1}$ Yong-Cheol Shin, ${ }^{1}$ Seong-Gyu Ko ${ }^{1}$
}

To cite: Cheon C, Park S, Park YL, et al. Sipjeondaebotang in patients with cancer with anorexia: a protocol for a pilot, randomised, controlled trial. BMJ Open 2016;6:e011212. doi:10.1136/bmjopen-2016011212

- Prepublication history and additional material is available. To view please visit the journal (http://dx.doi.org/ 10.1136/bmjopen-2016011212).

CC and SP contributed equally to this work.

Received 21 January 2016 Revised 8 March 2016 Accepted 12 April 2016

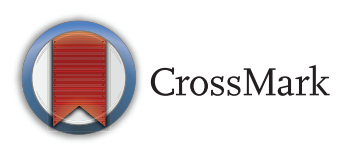

${ }^{1}$ Department of Korean Preventive Medicine, Graduate School, Kyung Hee University, Seoul, Republic of Korea

${ }^{2}$ Department of Preventive Medicine, College of Korean Medicine, Daejeon University, Daejeon, Republic of Korea

Correspondence to Professor Seong-Gyu Ko; epiko@khu.ac.kr

\section{ABSTRACT}

Introduction: Cancer-related anorexia is the loss of appetite or desire to eat in patients with cancer. Although treatments for cancer-related anorexia do exist, patients have sought complementary and alternative medicine including herbal remedies, due to safety concerns. Sipjeondaebo-tang is one among other popular herbal medicines that are beneficial to management of anorexia in Korea. The purpose of this study is to examine the feasibility for a full randomised clinical trial of Sipjeondaebo-tang for cancer-related anorexia.

Methods and analysis: This study is a randomised, double-blinded and placebo-controlled trial of Sipjeondaebo-tang. For the study, 40 patients with cancer, aged 20-80 years, who reported anorexia, will be recruited. The participants will receive either $3 \mathrm{~g}$ of Sipjeondaebo-tang or a placebo, 3 times a day for 4 weeks. The primary end point is a change in the anorexia/cachexia subscale (A/CS) of Functional Assessment of Anorexia/Cachexia Therapy (FAACT). The secondary end points include changes in the visual analogue scale (VAS) of appetite, cortisol and ghrelin. The outcomes will be measured on every visit. Each participant will visit once a week during 4 weeks.

Ethics and dissemination: The present study has been approved by the Institutional Review Board of the Dunsan Korean Medicine Hospital of Daejeon University (reference DJDSKH-15-03-2 (V.2.0)). The results will be disseminated in a peer-reviewed journal and scientific conference.

Trial registration number: NCT02468141; Pre-results.

\section{INTRODUCTION}

The cancer anorexia-cachexia syndrome (CACS) is a cascade reaction of appetite loss, malnutrition and weight loss, particularly muscle mass. ${ }^{1}$ This symptom is also called cancer-related or cancer-associated anorexia and appears among 15-40\% of patients with cancer. $^{2}$ It can be caused either by the cancer itself or by cancer treatment. As weight loss is associated with poor prognosis

\section{Strengths and limitations of this study}

- This is the first randomised controlled trial to examine the feasibility of Sipjeondaebo-tang for treatment of cancer-associated anorexia.

- Qi-deficiency syndrome and blood deficiency syndrome are used to evaluate general health status of patients with cancer reporting anorexia.

- Limitations of this study are its relatively smal sample size and the fact that it is a single-centre pilot trial.

and early death, management of cancerrelated anorexia is very important. ${ }^{3}$

Appetite stimulants such as corticosteroids (dexamethasone) and progestational agents (megestrol) are the first-line therapy for patients with anorexia. ${ }^{4}$ However, it has been reported that long-term and high dose administration of these agents causes symptoms related to adrenal suppression. Other agents including nutraceuticals, fluoxymesterone, pentoxifylline, hydrazine sulfate, dronabinol, cyproheptadine, eicosapentaenoic acid (EPA) and etanercept (Enbrel) show little or no benefit at all. ${ }^{5}$ Some patients seek to receive alternative safe and effective therapies such as herbal medicines for cancerrelated anorexia. ${ }^{6}$ Among these remedies, Sipjeondaebo-tang (SJDBT, also known as Juzen-taiho-to and Shi-Quan-Da-Bu-Tang, in Japanese and Chinese, respectively) has recently been found to have the potential effect of an antiemetic, as well as ameliorating cancer-induced anorexia and cachexia. $^{6} 89$

SJDBT is the third most frequently prescribed herbal medicine in Korean medicine clinics. $^{10}$ SJDBT was first introduced to Chinese medicine in the Chinese medical classic titled 'Taipinghuiminhejijufang (Prescriptions from the Great Peace Imperial Grace Pharmacy)', written by Chen Shiwen in $1107 .^{11}$ It includes a combination of 10 
herbs: Ginseng Radix Alba, Atractylodis Rhizoma Alba, Poria Sclerotium, Glycyrrhizae Radix, Angelicae Gigantis Radix, Cnidii Rhizoma, Rehmanniae Radix Preparata, Paeoniae Radix, Astragali Radix and Cinnamomi Cortex. $^{12}{ }^{13}$ SJDBT is prescribed to balance Yin and Yang by treating both qi-deficiency and blood deficient syndromes due to exhaustion. ${ }^{14}$ In practice, SJDBT is prescribed for weakness after illness, anorexia, night sweats, cold hands and feet, and anaemia. ${ }^{15}$

Although results of clinical studies on SJDBT for treating anorexia have been continuously published in medical journals, most are case reports or observational studies on the association between anorexia and nutritional conditions, and have a low level of evidence. ${ }^{16} 17$ Therefore, in this study, we rigorously designed a randomised, placebo-controlled clinical trial to examine the feasibility of a full randomised clinical trial of a pharmacological intervention, SJDBT, for managing anorexia in Korean patients with cancer.

\section{METHODS}

\section{Study design}

A randomised, double-blind, placebo-controlled trial will be conducted at the Dunsan Korean Medicine Hospital of Daejeon University in Daejeon, Republic of Korea. All patients fulfilling the eligibility criteria will be selected. The enrolled participants will be randomly allocated to two parallel groups: the SJDBT group and placebo group. Each participant will be examined for signs and symptoms of anorexia before and after taking the experimental drug. Figure 1 shows the schematic flow of the study. Protocol modifications are not expected. However, if it is necessary, any change in the protocol will be communicated to all the investigators, in a conference. All changes will be included in the final manuscript for journal submission.

\section{Recruitment}

Participants will be recruited through two routes. Patients who visit the trial site and meet the inclusion criteria will be recommended, by the physician in charge of the study, to participate in the trial. Detailed information on the trial including study period, purpose of study, inclusion and exclusion criteria, and intervention, is posted on a bulletin board at the hospital, and those patients who check the board will voluntarily visit the trial site.

\section{Participants}

\section{Inclusion criteria}

Participants will be included if they meet the following criteria: men and women aged 20-80 years; patients who have a histologically or cytologically confirmed solid tumour; individuals who are suffering from anorexia; patients within 1 month of completion of chemotherapy; those with no plan for additional chemotherapy or radiotherapy; patients with: anorexia visual analogue
Enrolment

Participant recruitment

Allocation

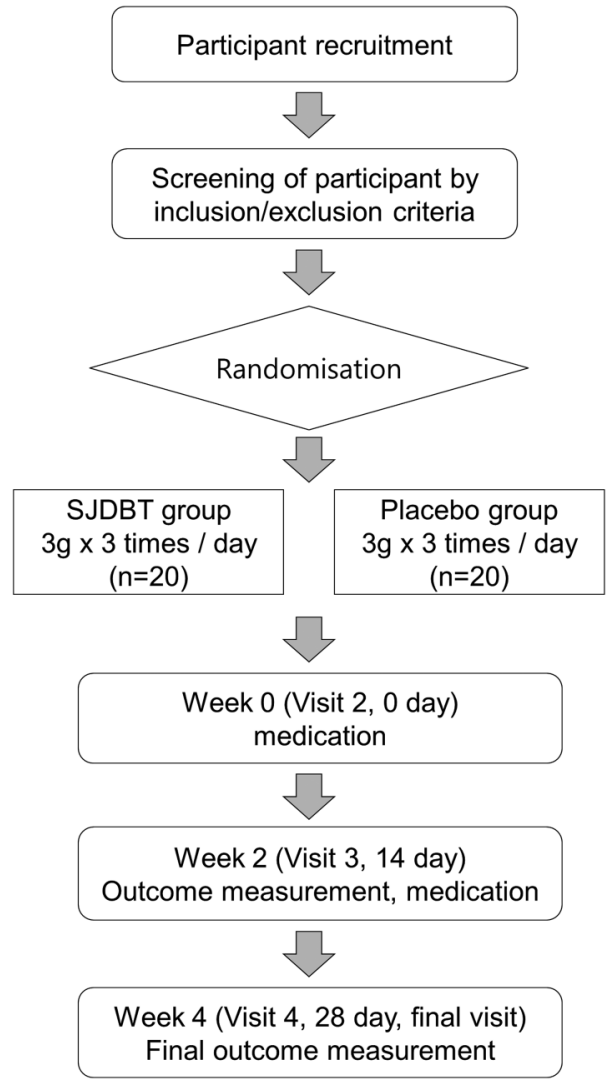

Figure 1 Study flow chart. SJDBT, Sipjeondaebo-tang.

scale (VAS) $\geq 40 / 100 \mathrm{~mm}$, qi-deficiency scale $\geq 30$ or blood deficiency scale $\geq 30$, neutrophils $\geq 1500 / \mu \mathrm{L}$, platelets $\geq 100000 / \mu \mathrm{L}$, total bilirubin of the maximum normal level or less $(1.2 \mathrm{mg} / \mathrm{dL})$,alanine transaminase, aspartate transaminase lower than twofold of the upper limit of normal, creatinine lower than 1.5-fold the upper limit of normal $(1.09 \mathrm{mg} / \mathrm{dL})$; and those who provide written informed consent for participation in the trial.

\section{Exclusion criteria}

The exclusion criteria are as follows: patients unable to intake orally; patients who have survived at least 5 years after cancer diagnosis; those with an Eastern Cooperative Oncology Group (ECOG) performance status score $>3$; patients with dementia, delirium and depression; patients who report more than seven points on the Numeric Pain Rating Scale; patients suffering from diseases, such as hypoadrenalism, that can influence appetite; patients who are taking palliative medicines such as megestrol acetate, corticosteroids and thalidomide; women of childbearing potential; and individuals judged inappropriate for the study.

\section{Participant withdrawal criteria}

Participants who meet the withdrawal criteria will be discontinued from the trial. The participants who are withdrawn after randomisation will be followed for outcomes. Use of any forbidden medication or treatment 
during the trial that could affect the study result; participant's withdrawal of consent; occurrence of a serious adverse event; detection of eligibility violations, occurrence of other significant protocol violations during the trial; and investigator's decision to terminate the process for the sake of the participant's health, will lead to withdrawal from the trial.

\section{Sample size}

The present study is a pilot study that examines the feasibility of a full randomised clinical trial of SJDBT for treating cancer-related anorexia and determines the effect size for further large-scaled studies. Thus, a total of 40 participants will be recruited for the study. Twenty participants will be allocated to the SJDBT group and another 20 to the placebo group.

\section{Randomisation and allocation}

Study participants who satisfy the eligibility criteria will be randomised using randomly generated numbers at an independent centre, Institute of Safety, Efficacy and Effectiveness Evaluation for Korean Medicine (ISEE), which is the contract research organisation (CRO) of this study. Block randomisation with block size of four will be performed. The participants in the present study will be assigned to one of two groups with an allocation ratio of 1:1. An opaque and sealed envelope containing the randomly generated numbers and a randomisation table will be maintained by the ISEE during the research period. The randomisation table placed in the opaque sealed envelope by the CRO will be opened according to standard operating procedure (SOP).

\section{Blinding}

A research assistant not involved in recruitment, intervention or assessment of outcomes, will prepare the envelopes. Investigators responsible for recruitment and assessment are not allowed to take part in the group allocation. Therefore, all the investigators, monitors and participants will be blinded for assignment of the study drugs. The experimental products will be produced and labelled using the randomisation numbers, at the Hanpoong Pharm \& Foods Co, Ltd, a pharmaceutical company. For this procedure, the random number table will be provided to the pharmaceutical company. The labelled experimental products will be provided to the trial site by the pharmaceutical company.

\section{Treatment protocol}

The participants will receive SJDBT or a placebo drug for 4 weeks. They will orally take $3 \mathrm{~g}$ of granules with water three times a day after meals for 4 weeks. The daily doses follow Ministry of Food and Drug Safety (MFDS) recommended dosage and administration of SJDBT. The participants will be required to return any remaining drug for calculating compliance. During the trial, the participants will be prohibited to receive other treatment for anorexia.

\section{Interventions}

Hanpoong Pharm \& Foods Co, Ltd, produces the SJDBT and placebo in accordance with Korea Good Manufacturing Practice (KGMP) standards. SJDBT used in this study is a dark brown-coloured granule. It is a compound of Cinnamomi Cortex $1.00 \mathrm{~g}$, Paeoniae Radix $1.00 \mathrm{~g}$, Atractylodis Rhizoma Alba $1.00 \mathrm{~g}$, Ginseng Radix Alba 1.00 g, Cnidii Rhizoma 1.00 g, Astragali Radix 1.00 g, Poria Sclerotium $1.00 \mathrm{~g}$, Rehmanniae Radix Preparata $1.00 \mathrm{~g}$, Angelicae Gigantis Radix $1.00 \mathrm{~g}$ and Glycyrrhizae Radix $0.5 \mathrm{~g}$. These raw materials will be extracted and concentrated to $3 \mathrm{~g}$ per dose. The placebo is made of lactose, corn starch and caramel colouring, and has appearance, shape, weight, taste and colour similar to SJDBT.

\section{Primary outcome measurement}

The primary outcome in the present study is the change in the anorexia/cachexia subscale (A/CS) of Functional Assessment of Anorexia/Cachexia Therapy (FAACT) between baseline (visit 2) and the end of study (visit 4) ${ }^{18}$ A Korean translation version of FAACT, which is produced by the Functional Assessment of Chronic Illness Therapy (FACIT) organisation, will be used. ${ }^{18}$ The FAACT is a clinical tool for assessing the general aspects in quality of life for patients with cancer in addition to specific anorexia-related concerns. Among a total of 39 items, it has a subscale of 12 items focusing on anorexia and cachexia. The FAACT will be measured by a trained investigator at every visit with SOPs.

\section{Secondary outcome measurement}

Secondary outcome measurements include changes in Functional Assessment of Cancer Therapy-General (FACT-G),${ }^{18}$ VAS of appetite, qi-deficiency and blood deficiency scale, ${ }^{19}$ weight and body mass index, and clinical laboratory tests such as adrenocorticotropic hormone $(\mathrm{ACTH})$, cortisol, ghrelin, interleukin 6, erythrocyte sedimentation rate and $\mathrm{C}$ reactive protein. The Korean translation version of the FACT-G is validated and qi-deficiency and blood deficiency scale will be translated by a Korean expert. ${ }^{2021}$ The study schedule is detailed in table 1.

\section{Safety outcomes}

All variables related to safety assessment, such as vital signs, physical examination, various laboratory test results including haematological test, biochemical test and urine test, and adverse events, will be documented on the case report form (CRF), at every visit.

\section{Statistical analysis}

Efficacy assessment

Statistical analyses will be performed for both the intention-to treat (ITT; all randomly assigned participants) and per-protocol (PP; participants completed the trial without any protocol deviations) data sets. The primary end point will be analysed using the ITT data set, and compared with the analysis, using the PP data set. The missing values will be imputed by the multiple 
Table 1 Study schedule of the SJDBT clinical trial (4 weeks)

\begin{tabular}{|c|c|c|c|c|}
\hline \multirow[b]{3}{*}{ Time point } & \multicolumn{4}{|c|}{ Study period } \\
\hline & \multirow{2}{*}{$\begin{array}{l}\text { Enrolment } \\
\text { Day }-7\end{array}$} & \multicolumn{3}{|c|}{ Treatment period } \\
\hline & & Day 0 & Day 14 & Day 28 \\
\hline Eligibility screen & $\mathrm{x}$ & & & \\
\hline Informed consent & $\mathrm{x}$ & & & \\
\hline Allocation & & $\mathrm{x}$ & & \\
\hline \multicolumn{5}{|l|}{ SJDBT } \\
\hline \multicolumn{5}{|l|}{ Placebo } \\
\hline Demographic characteristic & $\mathrm{x}$ & & & \\
\hline Physical examination & $\mathrm{x}$ & $\mathrm{x}$ & $\mathrm{x}$ & $\mathrm{x}$ \\
\hline Chest X-ray, electrocardiography & $x$ & & & \\
\hline Laboratory test & $\mathrm{x}$ & $\mathrm{x}$ & $\mathrm{x}$ & $\mathrm{x}$ \\
\hline VAS for anorexia & $\mathrm{x}$ & $\mathrm{x}$ & $\mathrm{x}$ & $x$ \\
\hline Qi-deficiency syndrome scale & $\mathrm{x}$ & & & $\mathrm{x}$ \\
\hline Blood deficiency syndrome scale & $\mathrm{x}$ & & & $\mathrm{x}$ \\
\hline FAACT scale & & $\mathrm{x}$ & $\mathrm{x}$ & $\mathrm{x}$ \\
\hline Weight and BMI & $\mathrm{x}$ & $\mathrm{x}$ & $\mathrm{x}$ & $x$ \\
\hline Compliance calculation & & & $\mathrm{x}$ & $\mathrm{x}$ \\
\hline Concomitant medication & $\mathrm{x}$ & $\mathrm{x}$ & $\mathrm{x}$ & $x$ \\
\hline Medical/drug use history & $x$ & $\mathrm{x}$ & $\mathrm{x}$ & $\mathrm{x}$ \\
\hline Adverse event & & & $\mathrm{x}$ & $\mathrm{x}$ \\
\hline
\end{tabular}

imputations. The continuous variables will be displayed as the mean $\pm \mathrm{SD}$, and the categorical variables will be displayed as the $\mathrm{n}(\%)$. The baseline characteristics will be compared by either an independent Student's $t$ test for continuous variables or the $\chi^{2}$ test (Fisher's exact test will be used when the expected value is $<5$ ) for the categorical data. For the primary outcome measurement, the mean differences between baseline and the end of treatment will be compared using an independent Student's $t$ test. Alternatively, Wilcoxon rank sum test will be used if the normality assumption is not satisfied for the continuous variables. The normality assumption will be tested using the Shapiro-Wilk test. A two sided, $5 \%$ or lower $\mathrm{p}$ value will be considered statistically significant. The present study has no plans for interim analysis.

\section{Safety assessment}

All participants will be required to report any adverse events they experience during the trial, at every visit. Adverse events will be documented in the CRFs. If the adverse event is severe and associated with the trial, the participant will be considered as withdrawn from the trial, and then the appropriate treatment will be given to him or her. Any loss caused by the trial will be reimbursed by insurance. For the safety assessment, blood cell count test, urinalysis and physical examination will be performed at visit 1,2 and 4 . The safety-related variables will be analysed using the ITT data set.

\section{Data and safety monitoring}

To maintain the quality of this trial, monitoring will be conducted by the ISEE. The institution participating in the trial will be monitored while this trial is in progress, using
SOPs. To improve data quality, range checks for data values and double data entry will be carried out. Suspected and unexpected serious adverse reactions will be reported to the institutional review board (IRB) and regulatory authorities in the Republic of Korea within $24 \mathrm{~h}$.

\section{Ethics and dissemination}

The present study has been approved by the IRB of the Dunsan Korean Medicine Hospital of Daejeon University (reference DJDSKH-15-03-2 (V.2.0)). The current protocol version is 2.0. All items from the WHO Trial Registration Data Set have been drawn (see online supplementary appendix 1). Written informed consent will be obtained from each participant prior to enrolment, by the investigator (see online supplementary appendix 2 ). The trial will be performed in compliance with the Helsinki Declaration and according to Good Clinical Practice as described by the Korea Food and Drug Administration.

The confidentiality of personal information will be protected. Each participant will be assigned a study identification number at enrolment. Throughout the trial, data will be handled by the study identification number. During and after the trial, all the records will remain secured in a locked cabinet or as password-protected computer files. Only investigators will retain the right to access the data. The results of the present study will be disseminated through scientific journals and a scientific conference presentation.

\section{DISCUSSION}

This study investigates the clinical efficacy and safety of SJDBT for cancer-related anorexia. In 2012, there were 
14.1 million new cancer cases and 8.2 million deaths from cancer, while 32.6 million people throughout the world lived with cancer. ${ }^{22}$ In Korea, cancer is also the leading cause of mortality, and researchers assume that this trend will continue for several years. ${ }^{23}$

Global Industry Analysts, Inc (GIA), estimate that the complementary and alternative medicine (CAM) market will reach 114 billion in dollars until $2015 .^{24}$ In this context, much CAM research on cancer has been conducted. However, most of the research consists of preclinical studies or studies on development of new drugs. ${ }^{25} 26$

Patients with cancer often suffer from anorexia, which is one of the major cancer-associated symptoms. If herbal medicine alleviates the symptom, it will contribute to improving quality of life in patients with cancer. SJDBT has been recognised as being a potentially effective herbal medicine for management of anorexia in patients with cancer and is a frequently prescribed herbal medicine in real practice in East Asian countries. However, there are few clinical trials investigating its efficacy and safety. Although there are clinical studies on SJDBT, the study participants are limited to hip surgery and haemodialysis patients. There has been no clinical trial on SJDBT for patients with cancer. ${ }^{27} 28$ Thus, in this study, we will evaluate the efficacy of SJDBT, an herbal medicine consisting of 10 herbs that are known to be beneficial to strengthen the body after illness and to treat anorexia. In practice, it is difficult to confirm whether or not the cause of anorexia is cancer. Thus the present study will recruit every patient with cancer who has anorexia, but exclude participants who have disease or symptoms that can influence on appetite, such as depression, pain and hypoadrenalism. For recruiting participants who are currently suffering from cancer, patients who have survived for at least 5 years after cancer diagnosis will be excluded.

Even though similar studies have already been published on cancer treatment using herbal medicines such as Shu Gan Jian Pi granules, Fuzheng Kang'ai decoction and Shen-Mai San, ${ }^{29-31}$ this study has its own significance. First, it is the first randomised controlled trial to investigate the efficacy of SJDBT for treatment of cancerassociated anorexia in Korea. Whereas the studies mentioned above focused on cancer-related fatigue, quality of life and survival itself, this study focuses on anorexia in patients with cancer and measures anorexia-related factors including both, patients' reported outcomes such as FAACT, and anorexia VAS and biomarkers such as ACTH, cortisol and ghrelin, to evaluate them objectively. Second, the study is the first trial protocol using qi-deficiency syndrome and blood deficiency syndrome to evaluate the general health status of patients with cancer reporting anorexia. The present study focuses on qi-deficiency and blood deficiency, which are both included in prescription criteria of SJDBT as well as anorexia. According to prescriptionology, SJDBT has its effectiveness in tonifying qi and blood, ${ }^{11}$ and thus it is clinically significant to evaluate the deficiencies of qi and blood. This trial may also provide scientific evidence for the efficacy of SJDBT for tonifying qi and blood and for increasing appetite. However, this study also has limitations, mostly stemming from its relatively small sample size and the fact that it is a singlecentre pilot trial. Considering recruitment feasibility, patients with cancer with different types and at different stages will be recruited, making the characteristics of each type and stage difficult to detect. Further studies are needed to assess the influence of the specific type and stage of cancer. Despite these limitations, this study would serve as a pilot report to plan further clinical trials on herbal medicine and cancerrelated anorexia.

Acknowledgements The authors wish to acknowledge Hanpoong Pharm \& Foods Co, Ltd, for providing investigational product support.

Contributors $\mathrm{CC}$ and SP wrote the initial manuscript and monitored this trial. $\mathrm{C}-\mathrm{WH}, \mathrm{YK}, \mathrm{YLP}, \mathrm{B}-\mathrm{HJ}$ and $\mathrm{Y}$-CS edited the first manuscript. S-GK conducted all the procedures for this protocol. All the authors have read and approved the final manuscript.

Funding This research was supported by a grant from the Korea Health Technology R\&D Project through the Korea Health Industry Development Institute (KHIDI), funded by the Ministry of Health \& Welfare, Republic of Korea (grant number HI12C1889). The management, analysis and reporting of the study will be conducted independently by the study investigators.

Competing interests None declared.

Patient consent Obtained.

Ethics approval The Institutional Review Board of the Dunsan Korean Medicine Hospital of Daejeon University approved the study (reference DJDSKH-15-03-2 (V.2.0)).

Provenance and peer review Not commissioned; externally peer reviewed.

Data sharing statement The results of the present study will be disseminated through scientific journal and scientific conference presentation.

Open Access This is an Open Access article distributed in accordance with the Creative Commons Attribution Non Commercial (CC BY-NC 4.0) license, which permits others to distribute, remix, adapt, build upon this work noncommercially, and license their derivative works on different terms, provided the original work is properly cited and the use is non-commercial. See: http:// creativecommons.org/licenses/by-nc/4.0/

\section{REFERENCES}

1. Fearon K, Strasser F, Anker SD, et al. Definition and classification of cancer cachexia: an international consensus. Lancet Oncol 2011;12:489-95.

2. Bruera E. Clinical management of anorexia and cachexia in patients with advanced cancer. Oncology 1992;49(Suppl 2):35-42.

3. Dewys WD, Begg C, Lavin PT, et al. Prognostic effect of weight loss prior to chemotherapy in cancer patients. Eastern Cooperative Oncology Group. Am J Med 1980;69:491-7.

4. Del Fabbro E. Current and future care of patients with the cancer anorexia-cachexia syndrome. Am Soc Clin Oncol Educ Book 2015;35:e229-37.

5. Yavuzsen T, Davis MP, Walsh D, et al. Systematic review of the treatment of cancer-associated anorexia and weight loss. J Clin Oncol 2005;23:8500-11.

6. Cheng KC, Li YX, Cheng JT. The use of herbal medicine in cancer-related anorexia/ cachexia treatment around the world. Curr Pharm Des 2012;18:4819-26.

7. Takeda $\mathrm{H}$, Sadakane $\mathrm{C}$, Hattori $\mathrm{T}$, et al. Rikkunshito, an herbal medicine, suppresses cisplatin-induced anorexia in rats via 5 -HT2 receptor antagonism. Gastroenterology 2008;134:2004-13. 
8. Lee JJ, Lee JJ. A phase II study of an herbal decoction that includes Astragali Radix for cancer-associated anorexia in patients with advanced cancer. Integr Cancer Ther 2010;9:24-31.

9. Choi YK, Jung KY, Woo SM, et al. Effect of Sipjeondaebo-tang on cancer-induced anorexia and cachexia in CT-26 tumor-bearing mice. Mediators Inflamm 2014;2014:736563.

10. Korea Food and Drug Administration (KFDA). Research on intake of Chinese medicine by Korean. KFDA 2007:36-9.

11. Park SG, Kim YK, Oh MS. Korean medicine prescription. Younglim Publisher, 2003.

12. KPIC. Korea Pharmaceutical Information Center. Secondary Korea Pharmaceutical Information Center, 2000. http://www.health.k

13. Jeon WY, Shin IS, Shin HK, et al. Gastroprotective effect of the traditional herbal medicine, Sipjeondaebo-tang water extract, against ethanol-induced gastric mucosal injury. BMC Complement Altern Med 2014;14:373.

14. Oh S, Cheon C, Park S, et al. The analysis of the recent research trend of Sipjeondabo-tang in Korea. J Soc Prev Korean Med 2014;18:113-23.

15. MFDS. MFDS portal site ezdrug. 2001. http://ezdrug.mfds.go.kr (accessed 3 Jul 2015)

16. Kim T, Kim HU, Song HJ. A case of gastric emphysema in anorexia nervosa presenting as acute gastric distension. Korean $\mathrm{J}$ Gastroenterol 2012;60:315-19.

17. Park JH, Lee TH, Cheon SL, et al. Severe acute liver and pancreas damage in anorexia nervosa. Korean J Gastroenterol 2009;54:257-60.

18. FACIT. FACIT.org. 2010. http://www.facit.org (accessed 8 Jul 2015).

19. Katsutoshi T. Kampo: Japanese-oriental medicine: insights from clinical cases. K.K. Standard McIntyre, 1993.

20. Lee $\mathrm{EH}$, Chun M, Kang S, et al. Validation of the Functional Assessment of Cancer Therapy-General (FACT-G) scale for measuring the health-related quality of life in Korean women with breast cancer. Jpn J Clin Oncol 2004;34:393-9.

21. Katsutoshi T. East-west medical science learning from the case. Koonja, 2005.

22. International Agency for Research on Cancer. GLOBOCAN 2012: Estimated Cancer Incidence, Mortality and Prevalence Worldwide in
2012. Secondary GLOBOCAN 2012: Estimated Cancer Incidence, Mortality and Prevalence Worldwide in 2012, 2012. http://globocan iarc.fr/Pages/fact_sheets_cancer.aspx

23. National Cancer Center. Cancer Facts \& Figures 2014. Ministry of Health \& Welfare, 2014:18-27.

24. Global Industry Analysts Inc. Alternative medicine. USA: Globa Industry Analysts, Inc., 2012.

25. Lam W, Jiang Z, Guan F, et al. The number of intestinal bacteria is not critical for the enhancement of antitumor activity and reduction of intestinal toxicity of irinotecan by the Chinese herbal medicine PHY906 (KD018). BMC Complement Altern Med 2014;14:490.

26. Saif MW, Li J, Lamb L, et al. First-in-human phase II trial of the botanical formulation PHY906 with capecitabine as second-line therapy in patients with advanced pancreatic cancer. Cancer Chemother Pharmacol 2014;73:373-80.

27. Kishida Y, Nishii T, Inoue T, et al. Juzentaihoto (TJ-48), a traditional Japanese herbal medicine, influences hemoglobin recovery during preoperative autologous blood donation and after hip surgery. Int $J$ Clin Pharmacol Ther 2009;47:716-21.

28. Nakamoto H, Mimura T, Honda N. Orally administrated Juzen-taiho-to/TJ-48 ameliorates erythropoietin (rHuEPO)-resistant anemia in patients on hemodialysis. Hemodial Int 2008;12(Suppl 2): S9-14.

29. Li C, Yang G, Yu M, et al. Effects of traditional Chinese medicine Shu Gan Jian Pi granules on patients with breast cancer and cancer-related fatigue: study protocol for a randomized controlled trial. Trials 2015;16:2261.

30. Yang XB, Wu WY, Long SQ, et al. Fuzheng Kang'ai decoction combined with gefitinib in advanced non-small cell lung with epidermal growth factor receptor mutations: study protocol for a randomized controlled trial. Trials 2015;16:11.

31. Lo LC, Chen CY, Chen ST, et al. Therapeutic efficacy of traditional Chinese medicine, Shen-Mai San, in cancer patients undergoing chemotherapy or radiotherapy: study protocol for a randomized, double-blind, placebo-controlled trial. Trials 2012;3232. 